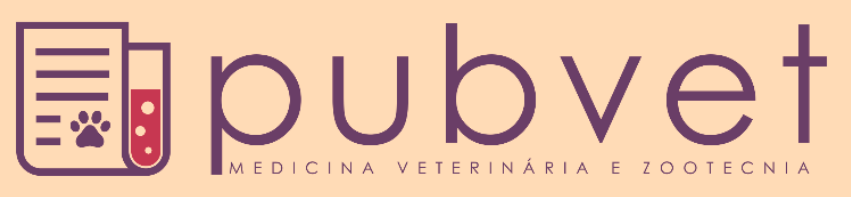

https://doi.org/10.31533/pubvet.v14n6a588.1-7

\title{
Síndrome glaucomatosa em Chihuahua: da hipertensão ocular ao glaucoma
}

\author{
Isadora Lobão Torres Santiago ${ }^{1}{ }^{\bullet}$, Raphael Willian Ponte Neres ${ }^{1}$, Stephany Ramos Pedrosa $^{10}$, \\ Maurício de Andrade Bilhalva ${ }^{2}{ }^{\circ}$, Tainá Ança Evaristo ${ }^{2}{ }^{\circ}$, Franciéli Ribeiro Silva ${ }^{2}$, Paula Priscila

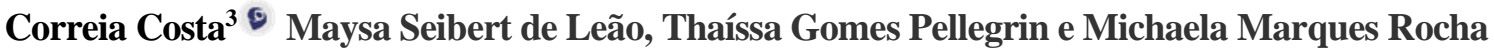 \\ ${ }^{I}$ Graduando (a) em Medicina Veterinária, Universidade Estadual do Ceará (UECE). \\ ${ }^{2}$ Graduando (a) em Medicina Veterinária, Universidade Federal de Pelotas (UFPel). \\ ${ }^{3}$ Doutora, docente no Departamento de Clínicas Veterinária (DCV), Hospital de Clínicas Veterinária (HCV), Universidade Federal de Pelotas. \\ Autor para correspondência, E-mail: paulapriscilamv@yahoo.com.br
}

\begin{abstract}
Resumo. O glaucoma é definido como a via final de um conjunto de enfermidades oftálmicas caracterizadas pela perda progressiva de sensibilidade e função, seguida de morte, das células ganglionares da retina e pela perda axonal do nervo óptico, com consequente redução do campo visual, conduzindo à cegueira, causado pelo aumento da pressão intraocular (PIO) considerada normal no cão entre 10 e $20 \mathrm{~mm} \mathrm{Hg}$. Nos animais pode ser classificado com relação a sua possível causa como primário, secundário ou congênito, com relação à aparência do ângulo de drenagem como glaucoma de ângulo aberto, de ângulo estreito ou de ângulo fechado, ou quanto a duração ou estágio da doença, como agudo, crônico ou absoluto. O diagnóstico é baseado nas manifestações clínicas associado com a utilização de tonometria, gonioscopia, ultrassonografia e eletrorretinografia. O tratamento é feito com base em fármacos que diminuem a PIO, podendo ser necessária associação com procedimentos cirúrgicos. As técnicas cirúrgicas objetivam diminuir a produção do humor aquoso, lesionando o corpo ciliar ou incrementando sua drenagem, através de vias alternativas.
\end{abstract}

Palavras chave: cão, cegueira, glaucoma, tratamento

\section{Glaucomatous syndrome in Chihuahua: from ocular hypertension to glaucoma}

\begin{abstract}
Glaucoma is defined as the final pathway of a set of ophthalmic diseases characterized by progressive loss of sensitivity and function, followed by death, of retinal ganglion cells and axonal loss of the optic nerve, with consequent reduction of visual field, leading to blindness, caused by increased intraocular pressure (IOP) considered normal in the dog between 10 and $20 \mathrm{~mm} \mathrm{Hg}$. In animals it may be classified as possible primary, secondary, or congenital cause with respect to the appearance of the drainage angle as openangle, narrow-angle, or angle-closure glaucoma, or as to the duration or stage of the disease, such as acute, chronic or absolute. The diagnosis is based on the clinical manifestations associated with the use of tonometry, gonioscopy, ultrasonography and electroretinography. The treatment is based on drugs that decrease IOP, and it may be necessary to associate with surgical procedures. Surgical techniques aim to reduce the production of aqueous humor, injuring the ciliary body or increasing its drainage, through alternative routes.
\end{abstract}

Keywords: dog, blindness, glaucoma, treatment 


\title{
Síndrome de glaucoma em Chihuahua: de hipertensión ocular a glaucoma
}

\begin{abstract}
Resumen. El glaucoma se define como la ruta final de un conjunto de enfermedades oftálmicas caracterizadas por la pérdida progresiva de la sensibilidad y la función, seguida de muerte de las células ganglionares de la retina, y por la pierda axonal del nervio óptico, con la consiguiente reducción del campo visual, conduciendo a la ceguera, causada por el aumento de la presión intraocular (PIO), considerada normal en perros entre 10 y $20 \mathrm{~mm}$ $\mathrm{Hg}$. En los animales se puede clasificar con respecto a su posible causa como primaria, secundaria o congénita, con respecto a la apariencia del ángulo de drenaje, como glaucoma de ángulo abierto, de ángulo estrecho o de ángulo cerrado, o según la duración y etapa de la enfermedad, como agudo, crónico o absoluto. El diagnóstico se base em las manifestaciones clínicas asociadas al uso de tonometría, gonioscopia, ultrasonido ocular y electrorretinografía. El tratamiento se basa em medicaciones que disminuyen la PIO, y puede ser necesario asociarlo como procedimientos quirúrgicos. Las técnicas quirúrgicas apuntan disminuir la producción del humor acuoso, lesionando el cuerpo ciliar o aumentando su drenaje por medio de rutas alternativas.
\end{abstract}

Palabras clave: perro, ceguera, glaucoma, tratamiento

\section{Introdução}

O glaucoma é definido como a via final de um conjunto de enfermidades oftálmicas caracterizadas pela perda progressiva de sensibilidade e função ocular, seguida de morte das células ganglionares da retina (CGR) e pela perda axonal do nervo óptico, com consequente redução do campo visual, conduzindo o indivíduo à cegueira (Whiteman et al., 2002; Whitley \& Gilger, 2003), causado pelo aumento da pressão intraocular (PIO) (Abrams, 2001; Brooks et al., 1997; Gelatt, 2003; Whiteman et al., 2002). A classificação do glaucoma canino é baseada na sua causa, na aparência do ângulo iridocorneal, zona anatômica resultante da união de 4 estruturas: córnea, esclera, íris e corpo ciliar; e na duração ou evolução da doença, que pode ser aguda ou crônica, sendo subdivido em glaucoma primário, secundário e congênito (Gelatt et al., 2008; Gelatt, 2003).

O glaucoma primário ocorre devido a uma anormalidade hereditária do ângulo irido-corneal, que gera aumento da PIO devido à obstrução da drenagem do humor aquoso, podendo ser classificado, ainda, como glaucoma primário de ângulo aberto, relatado com maior frequiência em cães da raça Beagle, Poodle miniatura, Poodle toy e Chihuahua (Gelatt et al., 2008; Plummer et al., 2008) e glaucoma primário de ângulo fechado, que não apresenta predisposição racial. Já o glaucoma secundário ocorre como consequência de alguma doença ocular pré-existente que cause obstrução física da drenagem do humor aquoso (Gelatt et al., 2008; Tinsley \& Betts, 1993), acontecendo, geralmente, de forma uni-lateral sem predisposição genética/racial. Com relação ao glaucoma congênito, é raro nos animais domésticos e, quando presente, inicia-se ao nascimento ou logo após (Slatter, 2007). É caracterizado pela elevação anormal da PIO associada a goniodisgenesia, ou seja, falha no no desenvolvimento da malha trabecular, condição também conhecida como displasia dos ligamentos pectinados (Gelatt et al., 2008). A goniodisgenesia é mais comum em cães de raças puras, como o Basset Hound e o Chihuahua.

Atualmente, o mercado pet vem crescendo e influenciando o cruzamento co-sanguíneo excessivo para formação de raças puras, devido ao seu maior valor de mercado. Tais cruzamentos vêm causando problemas congênitos e irreversíveis em algumas raças, dentre elas o chihuahua, que apresenta uma caixa encefálica reduzida em relação ao globo ocular, favorecendo o aparecimento de anomalias, como o glaucoma (CFMV, 2011).

O objetivo deste trabalho é fazer uma revisão de literatura a respeito da síndrome glaucomatosa, bem como explanar a respeito dos principais sinais clínicos, diagnóstico e tratamentos preconizados.

\section{Epidemiologia}

Considerados os menores cães do mundo, os Chihuahuas são naturais do continente americano, muito provavelmente da Cidade do México, tendo como ancestral um cão selvagem pequeno chamado Techichi, 
provavelmente por volta do século X. Entretanto, apenas em meados do século XX a raça foi oficializada, tendo seu primeiro exemplar aceito no American Kennel Club, registrado no ano de 1952. Entre as suas principais características raciais preconizadas, segundo a Confederação Brasileira de Cinofilia em 2010, estão o corpo compacto, de 15 a $22 \mathrm{~cm}$, pesando até $3 \mathrm{~kg}$, ou até $1 \mathrm{~kg}$ na variação mini da raça, com região craniana bem arredondada em forma de maçã, focinho curto, bochechas pouco desenvolvidas e muito secas e olhos grandes e redondos, bastante expressivos. Devido a essas características, ocorre aumento da predisposição à exoftalmia e à elevada PIO, devido ao globo ocular ser, por vezes, demasiadamente grande para ser comportado na cavidade ocular, principalmente na variação miniatura, tornando a raça uma das mais predispostas ao aparecimento de síndrome glaucomatosa.

Uma causa genética ou predisposição é suspeitada quando há uma prevalência aumentada de glaucoma em uma raça canina em particular. Então, além das análises de pedrigree, técnicas moleculares têm sido usadas para avaliar problemas de cunho genético que contribuem para o fenótipo da doença, seja diretamente por meio de problemas no ângulo irido-corneal, como relatado em cães da raça Shih-tzu e Shiba Inu, ou indiretamente, por meio de problemas na conformação anatômica e proporcional da cabeça e do globo ocular, como é relatado, atualmente, em Chihuahuas (Komáromy \& Petersen-Jones, 2015).

\section{Etiopatogenia e sinais clínicos}

A etiopatogenia do glaucoma ainda não é totalmente esclarecida, porém muitos autores consideram o aumento da PIO acima dos limites aceitáveis de 15 a $30 \mathrm{mmhg}$ para cães e gatos, como fator responsável pelas lesões oculares (Gelatt et al., 2008). Normalmente, os sinais clínicos demoram a aparecer, estando presentes apenas quando a PIO já se encontra bastante elevada, dificultando o tratamento (Rathbone-Gionfriddo, 1995). Dentre esses sinais, a presença de vasos episclerais ingurgitados, hiperemia conjuntival, edema de córnea (Figura 1) e a dor, expressa pelo animal ao esfregar os olhos nas patas ou em objetos, são os mais importantes, estando presentes em praticamente todos os casos relatados (Slatter, 2007).

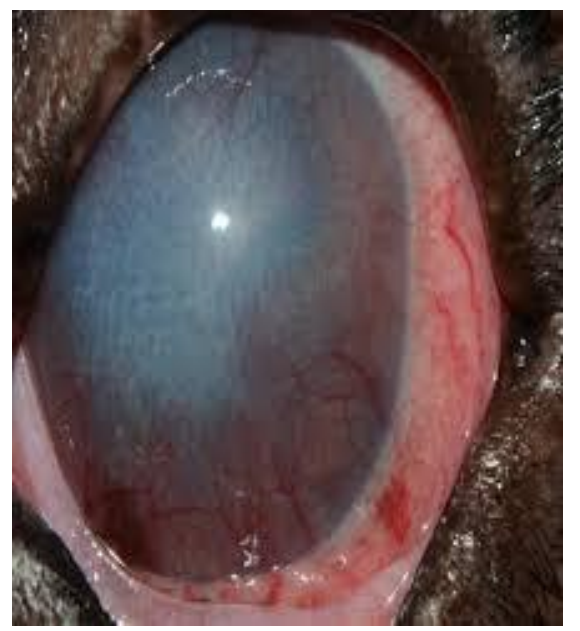

Figura 1. Macrofotografia de olho de cão portador de glaucoma, com notada neovascularização de vasos conjuntivais e episclerais, acompanhados de hiperemia conjuntival e edema difuso. Fonte: $w w w$.journals.ufrpe.br

Normalmente, quando a PIO excede 40 mmhg, observa-se midríase não responsiva, ou dilatação da pupila, e paralisia do músculo esfínter da íris (Gelatt, 2003; Miller et al., 1996) e com pressões acima de $60 \mathrm{mmhg}$ ocorre dano irreversível à retina e ao nervo óptico, levando o animal à cegueira em $24 \mathrm{a}$ 48h (Figura 2) (Koch \& Sykes, 2002).

A medida que ocorre o aumento da PIO, aumenta-se o tamanho do bulbo do olho (buftalmia), podendo levar ao desenvolvimento de megalocórnea, que, assim, torna-se mais exposta aos agentes externos e menos lubrificada pelo filme lacrimal, estando sujeita a ulcerações, ceratitites por exposição e perfurações (Figura 3) (Gelatt et al., 2008). O aumento da PIO pode, ainda, resultar em luxação de lente, secundária a buftalmia, e formação de catarata, estando relacionada, principalmente, às alterações na composição ou na taxa de formação do humor aquoso (Gelatt et al., 2008). Pode ocorrer, ainda, 
iridociclite, bloqueio pupilar, colapso da fenda ciliar, decorrente da ausência de tração nos ligamentos zonulares e opacificação da córnea (Slatter, 2007).

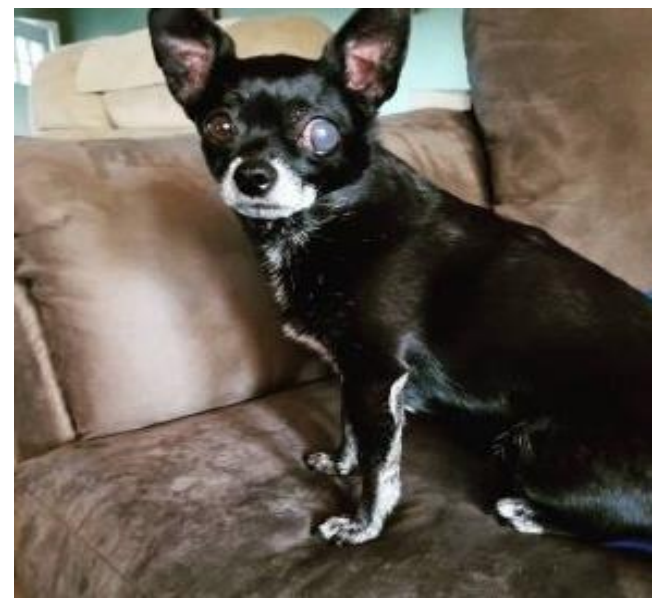

Figura 2. Macrofotografia de Chihuahua portador de glaucoma que evoluiu para cegueira e opacificação da córnea devido ao aumento exacerbado da PIO. Fonte: https://springsvet.com/meet-tinkerbell-ourcanine-glaucoma-patient/

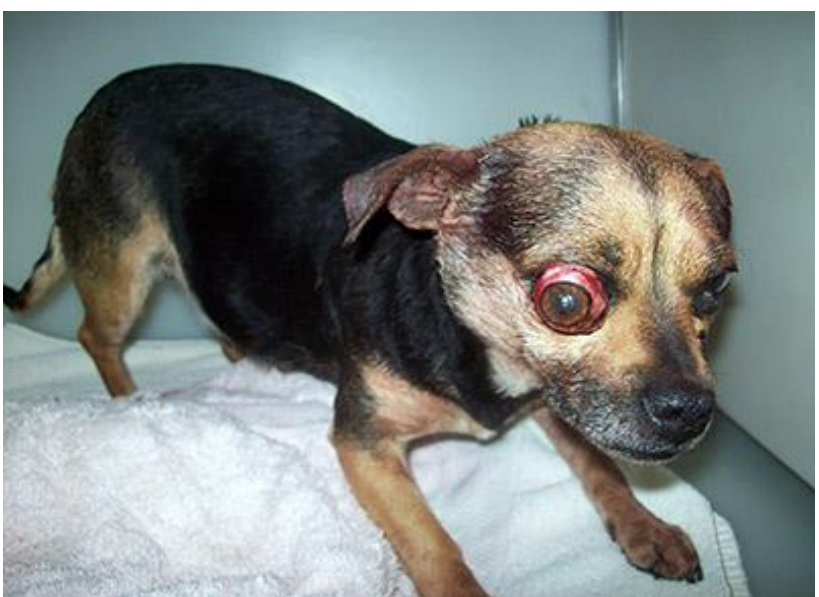

Figura 3. Macrofotografia de Chihuahua portador de glaucoma com grande aumento da PIO, formando a megalocórnea, neovascularização de vasos conjuntivais e episclerais, hiperemia conjuntival e midríase. Fonte: https://springsvet.com/meet-tinkerbell-our-canineglaucoma-patient/

\section{Exames complementares}

Os exames mais eficazes para a detecção do glaucoma são a tonometria, a gonioscopia e a oftalmoscopia. Há outros métodos de diagnósticos, como a ultrassonografia e a eletroretinografia (Silva, 2017). Na tonometria de aplanação, a PIO é deduzida a partir da força necessária para aplanar (achatar) uma área da córnea, apresentando o resultado em mmHg em um leitor. Faz-se necessário o uso do anestésico tópico antes do exame, já que a sonda entrará em contato com a córnea (McLellan et al., 2013). Na tonometria de rebote, os tonômetros medem a PIO através de uma pequena sonda de metal magnetizada e com ponta plástica. A sonda é projetada em direção a córnea e a velocidade na qual a sonda regressa ao dispositivo é convertida em sinais elétricos, a partir dos quais a PIO é calculada. Não é necessário o uso de colírio anestésico antes do procedimento (McLellan et al., 2013).

Na gonioscopia é possível avaliar o ângulo de drenagem da câmara anterior e com isso, melhor definir a conduta terapêutica, pois este procedimento permite a diferenciação entre os tipos de glaucoma aberto e fechado, assim como a mensuração do grau de obstrução do ângulo iridocorneano. A técnica deve ser sempre realizada em ambos os olhos (Slatter, 2007).

$\mathrm{Na}$ oftalmoscopia é possível avaliar o fundo do olho, para evidenciação de alterações como hiperreflexividade da área tapetal (adelgaçamento da rima neurorretiniana), atenuação dos vasos retinianos e escavações no disco óptico. Possui duas formas de realização: a oftalmoscopia direta é feita com o uso do oftalmoscópio e proporciona um maior detalhamento; a oftalmoscopia indireta pode ser feita com o uso de uma lente de 20 dioptrias e uma lanterna, no entanto, para sua realização, se faz necessária a dilatação pupilar, o que possibilita um maior campo de visão e um menor detalhamento. A presença de opacificações corneanas podem impedir a realização da oftalmoscopia em ambas as formas (Slatter, 2007).

A ultrassonografia é utilizada em casos em que há anormalidades que impeçam a visualização no interior do olho. Constitui-se em um método importante na avaliação de anormalidades do segmento posterior do globo ocular, através de uma sonda de $20 \mathrm{MHz}$ (para detalhar anomalias da parede ocular posterior e da interface vítreo-retiniana) e uma sonda de $10 \mathrm{MHz}$ (melhor para detecção de anomalias no humor vítreo, particularmente nas porções mais anteriores da cavidade) (Mendes et al., 2009).

A eletroretinografia é um exame de extrema importância no diagnóstico de doenças que cursam com alterações funcionais das células ganglionares da retina, pois detecta a resposta das células da retina a um estímulo luminoso (Oriá et al., 2004). 


\section{Diagnóstico e tratamento}

A avaliação da PIO é preconizada, principalmente, em animais que apresentem os olhos vermelhos. Entretanto, o diagnóstico do glaucoma é feito através dos sinais clínicos e da execução de exames (Gelatt et al., 2008; Slatter, 2007. O diagnóstico da síndrome glaucomatosa é dado pela avaliação da PIO, que deve estar aumentada, e pela degeneração do nervo óptico (Gelatt, 2003).

O principal objetivo da terapia do glaucoma é preservar a visão. Normalmente, o tratamento clínico proporciona alívio temporário, mas não controla a PIO à longo prazo, por isso em muitos casos, recomenda-se a intervenção cirúrgica (Silva, 2017).

Os procedimentos disponíveis são variáveis e são utilizados dependendo do estágio do glaucoma e da potencial visão do animal. As técnicas para diminuição da PIO podem induzir a diminuição da produção do humor aquoso (HA), ou podem ser empregadas técnicas para aumentar a drenagem de HA (Silva, 2017).

\section{Tratamento terapêutico}

No tratamento farmacológico existem os diuréticos osmóticos, utilizados em casos agudos de elevação da PIO ou no pré cirúrgico, reduzindo o gradiente osmótico entre vasos sanguíneos oculares e humores aquoso e vítreo (Gelatt et al., 2008). Os agentes agonista adrenérgicos, como a apraclonidina e o taratarato brimonidina diminuem a PIO, pois reduzem a produção de HA, tendo ação em humanos, coelhos e cães (Gosling et al., 2016). Os inibidores da anidrase carbônica (IAC) reduzem a secreção de HA ativo pelo epitélio ciliar e são utilizados topicamente (McLellan et al., 2013).

Os análogos das prostaglandinas são utilizados de forma tópica, onde são absorvidos e biotransformadas em prostaglandinas, incluindo a PGE2, com grande potencial para ativar o sistema adenilciclase, remodelando a matriz extracelular do músculo ciliar da íris e aumentando desta forma a drenagem do HA pela rota uveoescleral (Sagara et al., 1999).

\section{Tratamento cirúrgico}

Atualmente os procedimentos cirúrgicos em animais glaucomatosos podem ser recomendados, devido ao maior conhecimento da patogênese e a melhoria das técnicas cirúrgicas (Plumer, 2013; Plummer et al., 2008). A cirurgia no glaucoma tem como objetivo alterar a produção, drenagem do HA, ou uma combinação de ambos. O dano ao epitélio secretor do corpo ciliar leva a uma diminuição da produção de HA, enquanto a cirurgia de filtração fornece um caminho alternativo para saída de HA (Plumer, 2013; Plummer et al., 2008).

Apesar dos avanços nas técnicas cirúrgicas, ainda há complicações pós-operatórias e à longo prazo em cães, permanecendo com o dano ocular irreversível. Os procedimentos realizados em estágio final desta afecção, tais como enucleação, evisceração com prótese intra-escleral e ablação química, estão disponíveis para proporcionar conforto aos bulbos oculares cegos e dolorosos (Silva, 2017).

\section{Prognóstico}

Em animais, o diagnóstico histopatológico é uma ferramenta importante e frequentemente utilizada no diagnóstico e na prevenção do glaucoma. A avaliação de um globo ocular enucleado pode auxiliar na confirmação do diagnóstico para o controle da condição e determinar o prognóstico para o olho remanescente. As alterações usualmente observadas à microscopia são o adelgaçamento da córnea, esclera e coroide devido à buftalmia, perda da continuidade da membrana de Descemet, edema de córnea, atrofia de toda a úvea, morte de células ganglionares e atrofia da retina, além de escavação do nervo óptico (Olbertz et al., 2012). Muito embora avanços nas cirurgias venham aumentando as perspectivas no manejo do glaucoma, a terapia médica mantém-se como componente importante no controle da síndrome glaucomatosa (Ribeiro et al., 2007). A maioria das substâncias preconizadas foi concebida para diminuir a PIO. São classificadas, segundo seu mecanismo de ação, em agentes capazes de reduzir a produção do humor aquoso (agonistas adrenérgicos e inibidores da anidrase carbônica), agentes que aumentam a drenagem do humor aquoso (agentes colinérgicos e análogos das prostaglandinas) e aqueles que atuam nas duas situações da dinâmica desse fluido ocular (antagonistas adrenérgicos) (Ribeiro et al., 2007; Willis, 2004). 
Atualmente, o tratamento do glaucoma canino abrange apenas o controle da pressão intraocular, reduzindo a dor do paciente e controlando a perda visual. Progressivamente a função visual tende a piorar, pois não há nenhuma substância capaz de impedir a apoptose retiniana (Ribeiro et al., 2007). Esta condição torna o glaucoma uma afecção com prognóstico reservado em longo prazo, acarretando desconforto e perda visual importante, reforçando a necessidade de conhecimento dos mecanismos e dos agentes etiológicos envolvidos nos quadros secundários à uveíte.

\section{Considerações finais}

O glaucoma se destaca por ser uma doença cada vez mais diagnosticada, mas que ainda permanece como um desafio, uma doença oftálmica considerada muito grave e que acomete tanto seres humanos como animais em todas as idades. Suas consequências podem ser devastadoras, quase sempre apresentando riscos eminentes de cegueira, sendo estes mais significativos em animais, uma vez que a maioria dos pacientes é encaminhada ao oftalmologista muito tardiamente.

Devido a esse fato, associado a terapias médicas e cirúrgicas limitadas para o glaucoma na medicina veterinária, pacientes com glaucoma incontrolado ou crônico geralmente apresentam condições agudas, tais como olhos irreversivelmente cegos, aumentados de tamanho e predispostos a ceratites de exposição. Nesses casos, o objetivo da terapia é aliviar a dor ocular.

A maior difusão de técnicas em tonometria na prática veterinária, aliada a exames de alta tecnologia, como ultrassonografia, Doppler colorido e eletrorretinografia, permitirão que um maior número de pacientes com glaucoma seja diagnosticado ainda nos estágios iniciais da doença, minimizando assim os riscos de perda da visão.

\section{Referências bibliográficas}

Abrams, K. L. (2001). Medical and surgical management of the glaucoma patient. Clinical Techniques in Small Animal Practice, 16(1), 71-76. DOI: https://doi.org/10.1053/svms.2001.22809

Brooks, D. E., Garcia, G. A., Dreyer, E. B., Zurakowski, D., \& Franco-Bourland, R. E. (1997). Vitreous body glutamate concentration in dogs with glaucoma. American Journal of Veterinary Research, 58(8), 864.

Gelatt, K. N. (2003). Manual de oftalmologia veterinária. Editora Monole.

Gelatt, K. N., Brooks, D. E., \& Kallberg, M. E. (2008). The canine glaucomas. Essentials of Veterinary Ophthalmology, 2, 155-187.

Gosling, A. A., Kiland, J. A., Rutkowski, L. E., Hoefs, A., Ellinwood, N. M., \& McLellan, G. J. (2016). Effects of topical corticosteroid administration on intraocular pressure in normal and glaucomatous cats. Veterinary Ophthalmology, 19, 69-76. DOI: https://doi.org/10.1111/vop.12355

Koch, S. A., \& Sykes, J. (2002). Keratoconjunctivitis sicca. In Small animal ophthalmology secrets (pp. 57-60). Hanley \& Belfus.

Komáromy, A. M., \& Petersen-Jones, S. M. (2015). Genetics of canine primary glaucomas. Veterinary Clinics: Small Animal Practice, 45(6), 1159-1182. DOI: https://doi.org/10.1016/j.cvsm.2015.06.003

McLellan, G. J., Kemmerling, J. P., \& Kiland, J. A. (2013). Validation of the TonoVet ${ }^{\circledR}$ rebound tonometer in normal and glaucomatous cats. Veterinary Ophthalmology, 16(2), 111-118. DOI: https://doi.org/10.1111/j.1463-5224.2012.01038.x

Mendes, M. H., Betinjane, A. J., Cavalcante, A. F., Castanheira, V. R., Cheng, C., \& Carani, J. C. (2009). Estudo comparativo entre imagens ultra-sonográficas obtidas com sondas de $10 \mathrm{MHz}$ e $20 \mathrm{MHz}$ na avaliação de anormalidades do segmento posterior do globo ocular. Revista Brasileira de Oftalmologia, 68(5), 291-295.

Miller, P. E., Nelson, M. J., \& Rhaesa, S. L. (1996). Effects of topical administration of 0.5\% apraclonidine on intraocular pressure, pupil size, and heart rate in clinically normal dogs. American Journal of Veterinary Research, 57(1), 79.

Olbertz, L., Perlmann, E., \& Ferreira, F. M. (2012). Achados histopatológicos do glaucoma em cães e gatos. Clínica Veterinária, 99, 66-76.

Oriá, A. P., Lázaro Júnior, L. P., Honsho, C. S., Dórea Neto, F. A., \& Laus, J. L. (2004). Considerations 
about electroretinography in dogs. Ciência Rural, 34(1), 323-328. DOI: http://dx.doi.org/10.1590/S0103-84782004000100053

Plumer, C. E. (2013). The Canine glaucomas. In K. N. Gelatt, B. C. Gilger, \& T. J. Kern (Eds.), Veterinary Ophthalmology, II (pp. 1050-1145). Wiley \& Sons.

Plummer, C. E., Källberg, M. E., Gelatt, K. N., Gelatt, J. P., Barrie, K. P., \& Brooks, D. E. (2008). Intranictitans tacking for replacement of prolapsed gland of the third eyelid in dogs. Veterinary Ophthalmology, 11(4), 228-233. DOI: https://doi.org/10.1111/j.1463-5224.2008.00630.x

Rathbone-Gionfriddo, J. (1995). Recognizing and managing acute and chronic cases of glaucoma. Veterinaria e Medicina, 90, 265-275.

Ribeiro, A. P., Martins, B. da C., \& Laus, J. L. (2007). Síndrome glaucomatosa em cães: parte 2. Ciência Rural, 37(6), 1828-1835. DOI: https://doi.org/10.1590/S0103-84782007000600054

Sagara, T., Gaton, D. D., Lindsey, J. D., Kaufman, P. L., \& Weinreb, R. N. (1999). Topical prostaglandin F2 $\alpha$ treatment reduces collagen types I, III, and IV in the monkey uveoscleral outflow pathway. Archives of Ophthalmology, 117(6), 794-801. DOI: 10.1001/archopht.117.6.794

Silva, T. C. (2017). Glaucoma em cães e gatos: revisão de literatura e estudo restropectivo. Trabalho de Conclusão de Curso. Graduação em Medicina Veterinária. Faculdade de Veterinária. Universidade Federal do Rio Grande do Sul, Porto Alegre, Brasil. 49 p.

Slatter, D. H. (2007). Manual de cirurgia de pequenos animais. Manole.

Tinsley, D. M., \& Betts, D. M. (1993). Glaucoma: past and present management techniques. Iowa State University Veterinarian, 55(1), 10.

Whiteman, A. L., Klauss, G., Miller, P. E., \& Dubielzig, R. R. (2002). Morphologic features of degeneration and cell death in the neurosensory retina in dogs with primary angle-closure glaucoma. Journal of the American Veterinary Medical Association, 63(2), 257-261. DOI: https://doi.org/10.2460/ajvr.2002.63.257

Whitley, R. D., \& Gilger, B. C. (2003). Doenças e cirurgia da córnea e esclera. In K. N. Gelatt (Ed.), Manual de oftalmologia veterinária (pp. 125-164). Manole.

Willis, A. M. (2004). Ocular hypotensive drugs. Veterinary Clinics: Small Animal Practice, 34(3), $755-$ 776. DOI: 10.1016/j.cvsm.2004.02.001

Recebido: 20 de dezembro, 2020.

Aprovado: 18 de abril, 2020.

Disponível online: 11 julho, 2020.

Licenciamento: Este artigo é publicado na modalidade Acesso Aberto sob a licença Creative Commons Atribuição 4.0 (CC-BY 4.0), a qual permite uso irrestrito, distribuição, reprodução em qualquer meio, desde que o autor e a fonte sejam devidamente creditados. 